\title{
Ursolic Acid Improves Intestinal Damage and Bacterial Dysbiosis in Liver Fibrosis Mice
}

\author{
Si-Zhe Wan', Cong Liu', Chen-Kai Huang, Fang-Yun Luo and Xuan Zhu* \\ Department of Gastroenterology, The First Affiliated Hospital of Nanchang University, Nanchang, China
}

Liver fibrosis is a reversible process of extracellular matrix deposition or scar formation after liver injury. Intestinal damage and bacterial dysbiosis are important concomitant intestinal changes in liver fibrosis and may in turn accelerate the progression of liver fibrosis through the gut-liver axis. RhoA, an important factor in the regulation of the cytoskeleton, plays an important role in intestinal damage. We investigated the effects of ursolic acid (UA), a traditional Chinese medicine with anti-fibrotic effects, on intestinal damage and bacterial

\section{OPEN ACCESS}

Edited by:

David Sacerdoti,

University of Verona, Italy

Reviewed by:

Rocchina Colucci,

University of Padua, Italy

Xufeng Tao,

Dalian University of Technology

(DUT), China

${ }^{*}$ Correspondence:

Xuan Zhu

jyyfyzx@163.com

tThese authors have contributed equally to this work

Specialty section:

This article was submitted to

Gastrointestinal and

Hepatic Pharmacology,

a section of the journal

Frontiers in Pharmacology

Received: 16 May 2019 Accepted: 15 October 2019

Published: 01 November 2019

Citation:

Wan S-Z, Liu C, Huang C-K, Luo F-Y and Zhu X (2019) Ursolic Acid

Improves Intestinal Damage and Bacterial Dysbiosis in Liver Fibrosis Mice.

Front. Pharmacol. 10:1321. doi: 10.3389/fphar.2019.01321 disorder through the RhoA pathway. UA treatment reduced intestinal damage by inhibiting the inflammatory factor TNF- $\alpha$ and increasing the expression of tight junction proteins and antibacterial peptides to protect the intestinal barrier. Moreover, the corrective effect of UA on bacterial dysbiosis was also confirmed by sequencing of the 16S rRNA gene. Potential beneficial bacteria, such as the phylum Firmicutes and the genera Lactobacillus and Bifidobacterium, were increased in the UA group compared to the $\mathrm{CCl}_{4}$ group. In liver fibrosis mice with RhoA inhibition via injection of adeno-associated virus, the liver fibrosis, intestinal damage, and flora disturbances were improved. Moreover, UA inhibited the expression of RhoA pathway components. In conclusion, UA improves intestinal damage and bacterial dysbiosis partly via the RhoA pathway. This may be a potential mechanism by which UA exerts its anti-fibrotic effects and provides effective theoretical support for the future use of UA in clinical practice.

Keywords: liver fibrosis, intestinal damage, bacterial dysbiosis, RhoA, ursolic acid

\section{INTRODUCTION}

Liver fibrosis is a reversible process of extracellular matrix (ECM) deposition or scar formation after liver injury caused by various factors, such as viruses, schistosomiasis, and alcohol (Lee et al., 2015; Seki and Brenner, 2015). Hepatic stellate cell (HSC) to myofibroblast (MFB) transformation is recognized as the central event in the development of liver fibrosis (Lee et al., 2015). The normal liver architecture and functions are disrupted in the liver in the state of liver fibrosis, and continuous development can eventually develop into cirrhosis and even liver cancer, which greatly threatens human health (Thompson et al., 2015; Affo et al., 2017). Liver transplantation is currently the only effective treatment once fibrosis develops into end-stage liver disease but is limited by the high cost and the shortage of donors and is thus unable to fully meet the needs of patients. Liver fibrosis is an early pathological feature of chronic liver disease due to its reversible characteristics; therefore, the development of anti-fibrotic treatments is of great significance. There is a special anatomical positional relationship between the liver and the gut; the portal vein-collected 
venous blood from the intestines provides $75 \%$ of the blood supply to the liver. The multiple physiological processes that are dependent on the reciprocal interaction between the liver and the intestines highlight the critical functional relationship between these organs. Therefore, some pathophysiological changes during liver fibrosis, such as impairment of the immune system, inflammation, and bile acid metabolism disorders, affect the intestinal tract through the gut-liver axis, causing intestinal damage and destruction of the intestinal barrier (Palma et al., 2007; Assimakopoulos et al., 2012; Kalaitzakis, 2014). The intestinal barrier acts as an important physical barrier to the intestine. Once this barrier breaks, it cannot prevent contaminants in the intestinal cavity from entering the blood and inducing liver and systemic lesions.

The human gastrointestinal tract hosts 10 100 trillion bacteria, including approximately 500 1,500 different bacterial species (Lozupone et al., 2012). The composition of the bacteria varies by individual, age, gender, and diet (Turnbaugh et al., 2009; Claesson et al., 2012). In recent years, many studies have confirmed that the intestinal microbiota participates in many activities in the body, such as metabolic homeostasis, immunomodulation, and inflammation (Maynard et al., 2012; Tremaroli and Backhed, 2012). As research has progressed, changes in the microbiota have been found in diabetes, arthritis, kidney disease, and atherosclerotic cardiovascular disease (Garidou et al., 2015; Jie et al., 2017; Ticinesi et al., 2018; Doonan et al., 2019).

The intestinal microbiota also undergoes dysbiosis during liver cirrhosis, which is often accompanied by a decrease in beneficial bacteria and an increase in harmful bacteria (Fouts et al., 2012; Shackel et al., 2014). Bacterial endotoxin, known as lipopolysaccharide (LPS), is found in the outer membrane of gram-negative bacteria; once LPS reaches the liver through a gap in the intestinal barrier, it is identified by the immune system through the recognition of pathogen-associated molecular patterns (PAMPs) (Kumar et al., 2011), which then induce the release of proinflammatory cytokines (Akira et al., 2006) and in turn accelerates the development of liver fibrosis.

Ursolic acid (UA) is a natural pentacyclic triterpenoid derived from various plants, including apples, basil, cranberries, and peppermint, and it has been reported to possess many biological activities, including anti-oxidative, anti-inflammatory, anti-ulcer, antibacterial, antiviral, anti-tumor, anti-obesity, hypoglycemic, antihypertensive, lipid-lowering, and liver protection properties (Ikeda et al., 2007; Wang et al., 2011; Wu et al., 2011; Kim et al., 2015; Cargnin and Gnoatto, 2017). A previous study showed that UA could reverse liver fibrosis by inhibiting the activation of HSCs in vivo and in vitro (He et al., 2015; Wang et al., 2011). However, the improvement of intestinal and microbiota dysbiosis by UA and the mechanisms involved are not clearly defined in liver fibrosis. RhoA, an important factor regulating the cytoskeleton by participating in actin stress fiber formation and myosin contraction (Asanuma et al., 2006; Kim et al., 2006), is involved in the integrity of the intestinal barrier (Tong et al., 2013) and has been shown to be associated with a variety of digestive diseases (Citalan-Madrid et al., 2017; Li et al., 2018). Therefore, in the present study, we aimed to determine the effects of UA on intestinal damage and microbiota dysbiosis in $\mathrm{CCl}_{4}$ induced liver fibrosis mice.

\section{MATERIALS AND METHODS}

\section{Experimental Animal Model and Design}

All wild-type (WT) C57BL/6 mice, obtained from the Department of Laboratory Animal Science of Nanchang University, were used for experiments. Mice were breed in an environment with a 12:12-h light/dark cycle, a room temperature of $22 \pm 2^{\circ} \mathrm{C}$, and $55 \pm 5 \%$ humidity. WT mice weighing 20 to $30 \mathrm{~g}$ were randomly divided into five groups as follows ( $n=8$ /group) (Figure 1): a control group was treated with olive oil $(2 \mathrm{ml} / \mathrm{kg}$ ) by gavage twice a week for 8 weeks (control group); liver fibrosis mice were induced by gavage of carbon tetrachloride $\left(\mathrm{CCl}_{4}\right)(20 \%$ olive oil dilution, $2 \mathrm{ml} / \mathrm{kg}$ ) twice a week for 8 weeks $\left(\mathrm{CCl}_{4}\right.$ group); mice were randomly selected and treated. One group of mice that was gavaged with $\mathrm{CCl}_{4}$ for 4 weeks was then gavaged simultaneously with UA (40 mg/kg/day) for another 4 weeks (UA group). A group of mice received adeno-associated virus (AAV) via tail vein injection for 1 week to inhibit RhoA and then received $\mathrm{CCl}_{4}$ gavage twice a week for 8 weeks (RhoAi group) (Supplementary Figure 1). All procedures were performed according to the National Institutes of Health Guide for the Care and Use of Laboratory Animals. The experimental protocol was approved

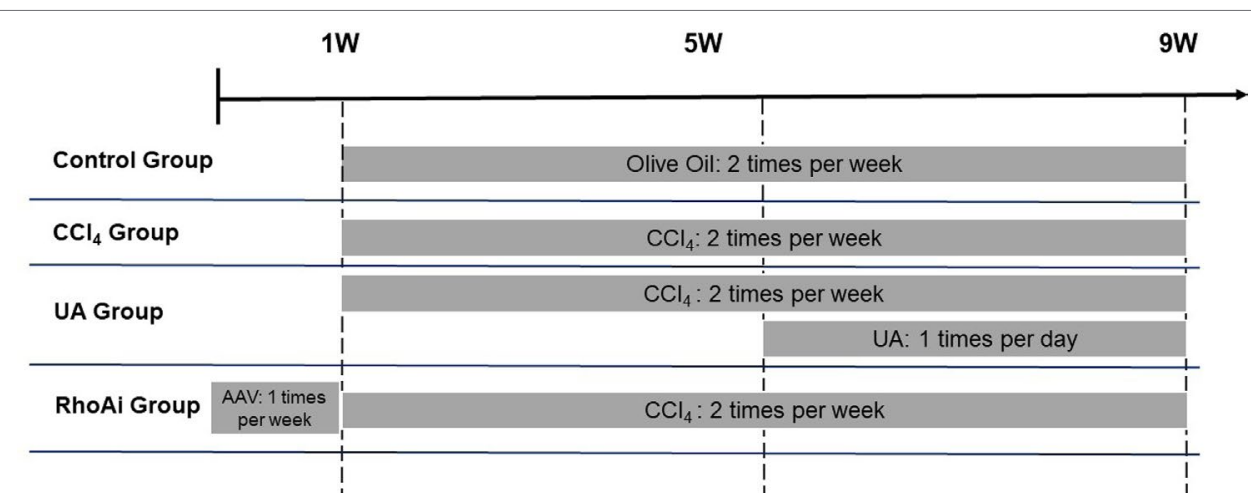

FIGURE 1 | Flow diagram depicting the treatment of mice in all groups. 
by the Animal Care and Use Committee of The First Affiliated Hospital of Nanchang University (Nanchang, China).

\section{Blood Index Test}

An automatic biochemical analyzer was used to detect alanine aminotransferase (ALT), total bilirubin (TBIL), aspartate aminotransferase (AST), and triglyceride (TG) (Department of Clinical Laboratory, The First Affiliated Hospital of Nanchang University, China). The LPS content of serum was estimated spectrophotometrically using commercial diagnostic kits purchased from Elabscience (China).

\section{Histological Analysis}

Liver and gut samples were fixed in $4 \%$ paraformaldehyde and cut into $5-\mu \mathrm{m}$ sections for staining with hematoxylin and eosin (H\&E), Masson's trichrome staining, immunohistochemistry (IHC), TdT-mediated dUTP nick-end labeling (TUNEL), and immunofluorescence. H\&E was used to observe the inflammatory cell infiltration of the liver and gut. We randomly selected five visual fields for observation, scored liver fibrosis using the METAVIR scoring system (Poynard et al., 1997) (Table 1), and evaluated intestinal mucosal damage using the Chiu scoring system (Chiu et al., 1970) (Table 2). IHC was used to reflect the expression site and expression intensity of related proteins. Liver fibrosis was estimated by Masson's trichrome staining. Liver section dual-immunofluorescence was used for the simultaneous observation of apoptosis of hepatocytes and expression of a-SMA (Abcam, Cat. 5694, USA). Specimens incubated with the antibody were observed and photographed by confocal microscopy.

TABLE 1 | METAVIR-based liver fibrosis scoring system.

\begin{tabular}{ll}
\hline Fibrosis grade & Comment \\
\hline F0 & $\begin{array}{l}\text { No fibrosis. } \\
\text { The fiber area of the portal area is enlarged, } \\
\text { but no septa are formed. }\end{array}$ \\
F2 & $\begin{array}{l}\text { The fiber area of the portal area is enlarged, } \\
\text { and a few septa are formed. }\end{array}$ \\
F3 & Numerous septa are formed without cirrhosis. \\
F4 & Cirrhosis. \\
\hline
\end{tabular}

F, fibrosis.

TABLE 2 | Chiu-based intestinal mucosal injury scoring system.

\begin{tabular}{ll}
\hline Grade & Comment \\
\hline 0 & $\begin{array}{l}\text { Normal intestinal mucosa. } \\
\text { Widening of the subepithelial space of the villus accompanied by } \\
\text { capillary congestion. } \\
2\end{array}$ \\
Extension of the subepithelial space with moderate lifting of the \\
epithelial layer from the lamina propria.
\end{tabular}

\section{Construction and Injection of Vectors for RhoA Inhibition}

To inhibit RhoA in vivo, AAV type 9 expressing a short hairpin RNA (shRNA) directed at RhoA (AAV-shRhoA) was injected into mice through the tail vein. The plasmid was designed with green fluorescent protein (GFP) to serve as a carrier for shRNA. The titer of the final AAV-shRhoA was $3.5 \times 10^{12}$ viral particles/ $\mathrm{ml}$ in phosphate-buffered saline (PBS).

\section{Gut Microbiota Analysis}

Genomic DNA was extracted from the feces of mice using a stool DNA kit (Omega, China) according to the manufacturer's instructions, and purity was verified by $1 \%$ agarose gel electrophoresis (BioFROXX, China). Primers (338F 5'-ACTCCTACGGGAGGCAGCAG-3' and 806R 5' -GGACTAC HVGGGTWTCTAAT- $3^{\prime}$ ) were used to amplify the bacterial V3-V4 region of the $16 \mathrm{~S}$ rRNA gene. Pyrosequencing of the polymerase chain reaction (PCR) products was performed on an Illumina MiSeq Instrument (Majorbio, China). Alpha diversity (Shannon and Chaol index), beta diversity, and community composition were calculated. Gut microbiota functional prediction was performed to generate the operational taxonomic unit (out) abundance table using PICRUSt. The Cluster of Orthologous Groups (COG) of proteins and Kyoto Encyclopedia of Genes and Genomes (KEGG) database were compared through the corresponding greengene id of each OTU to obtain the COG family and KEGG Orthology information corresponding to the OTU.

\section{Western Blot Analysis}

The liver and ileal tissues were homogenized in radioimmunoprecipitation assay (Solarbio, cat. R0020, China) buffer with phenylmethanesulfonyl fluoride (Cell Signaling Technology, cat. 8553, USA). The concentration of total protein was determined using a bicinchoninic acid (BCA) assay kit (Tiangen, Beijing, China). Equal quantities of protein extracts were resolved via $6-2 \%$ sodium dodecyl sulfate-polyacrylamide gel electrophoresis and electrophoretically transferred to polyvinylidene fluoride membranes. The membranes were blocked in 5\% non-fat milk and subsequently incubated overnight at $4^{\circ} \mathrm{C}$ with primary antibodies. Then, a secondary horseradish peroxidase-conjugated anti-rabbit or anti-mouse IgG antibody (ZSGB-BIO, China) was applied, and images were developed via an enhanced chemiluminescence (ECL) detection kit (Thermo Fisher Scientific, USA).

\section{Quantitative Real-Time Polymerase Chain Reaction Analysis}

Liver or ileal tissue was homogenized in $1 \mathrm{ml}$ of TRIzol (Life Technologies, USA), and total RNA was extracted. The integrity of RNA was verified via agarose gel electrophoresis, and RNA was converted to cDNA using a FastQuant RT kit (Tiangen, cat. KR10602, China). For qRT-PCR, SuperReal PreMix Plus (Tiangen, cat. SYBR Green, China) was used to determine the quantitative expression of RNA. The number of amplification cycles was 41 . 
A
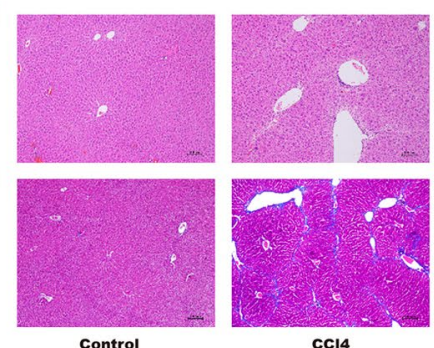

E
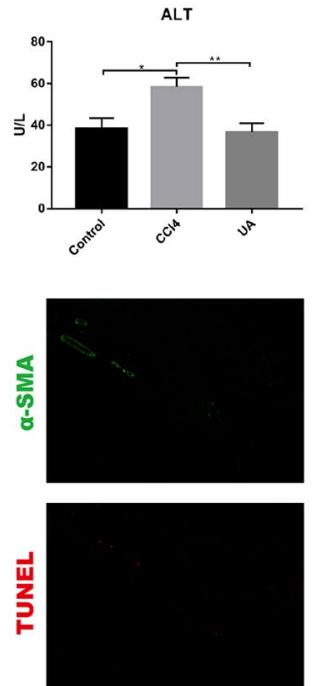

$\bar{a}$
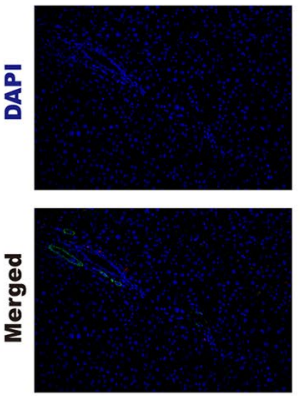

Control

G
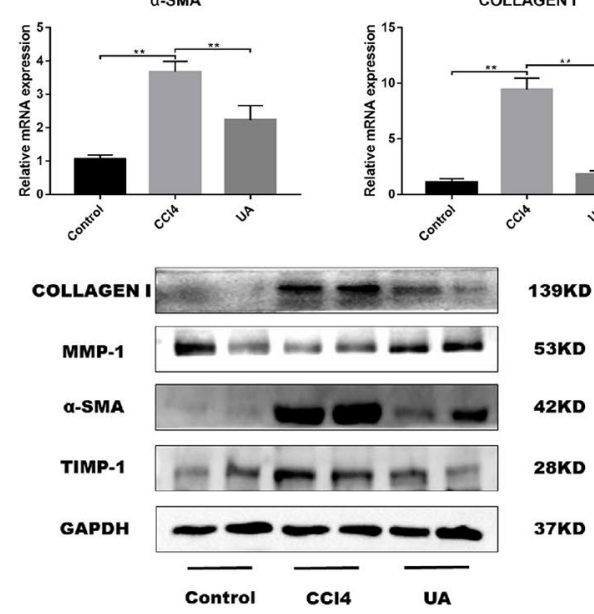
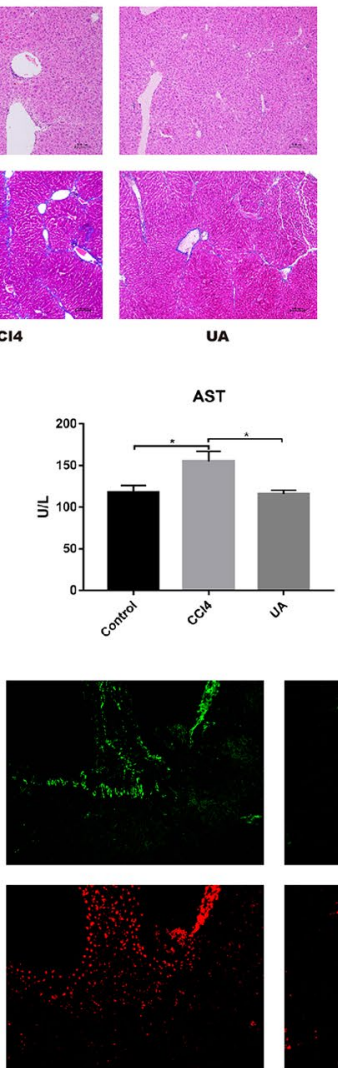

c
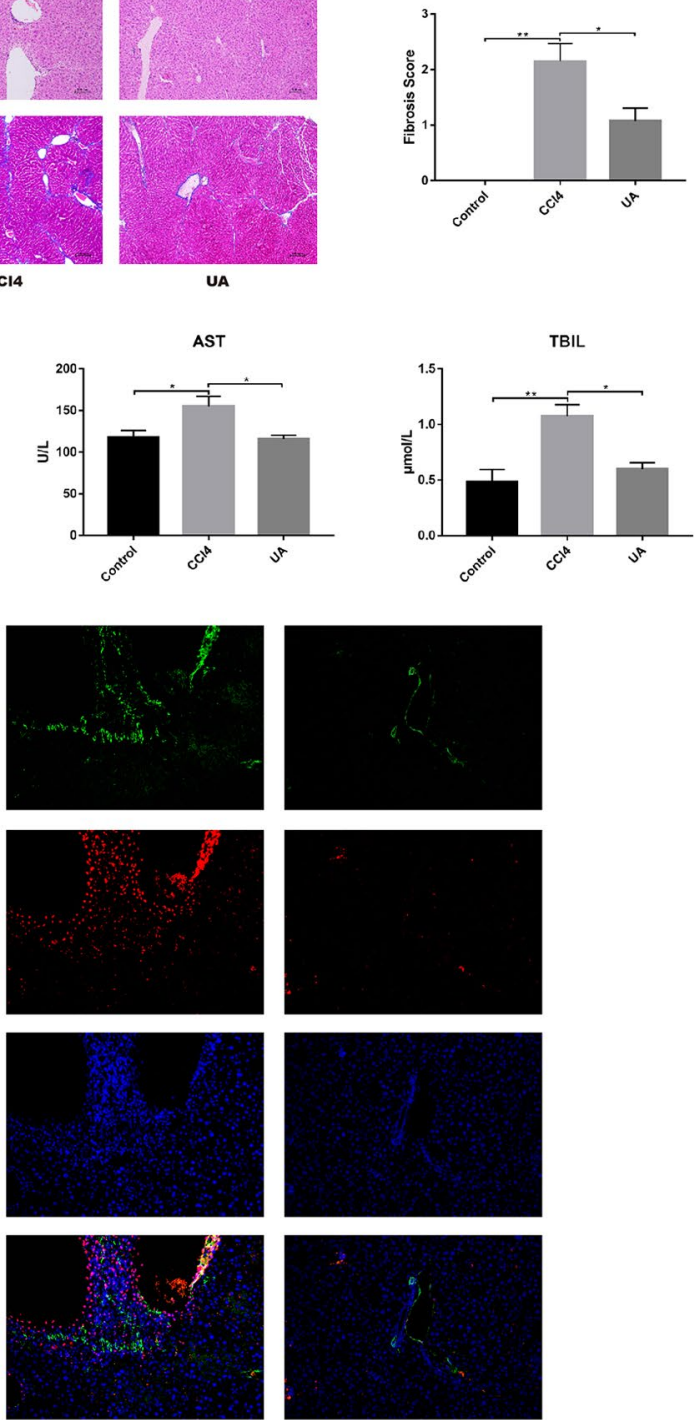

CCI4
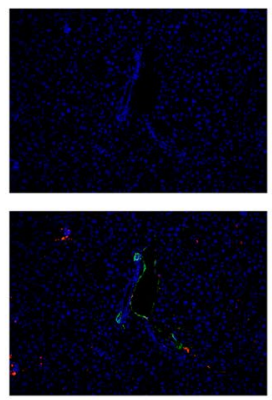

UA
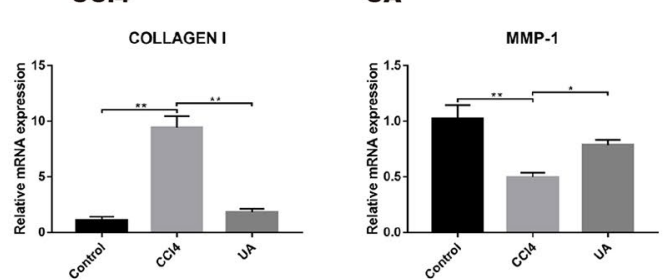

D
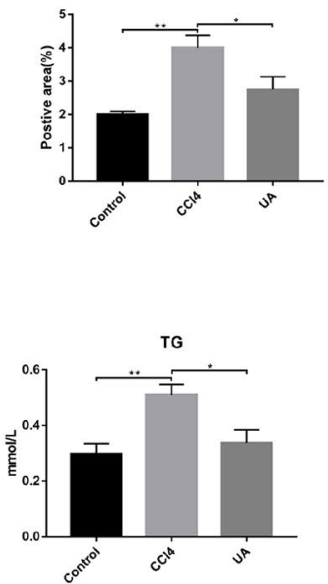

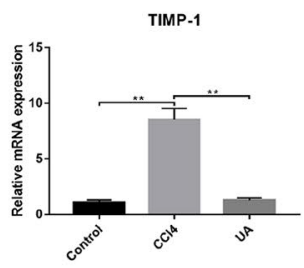

FIGURE 2 | Continued 
FIGURE 2 | Effect of UA on $\mathrm{CCl}_{4}$-induced liver injury and fibrosis. (A) Hematoxylin and eosin (H\&E) staining (100x magnification). (B) Masson's trichrome staining (100x magnification). (C) Morphometrical analysis for fibrotic score. (D) Semi-quantitative determination of fibrosis by Masson's trichrome staining. (E) ALT, AST, and TBIL levels in mouse serum. (F) Dual-immunofluorescence staining of liver sections from control group or $\mathrm{CCl}_{4}$ group or UA group stained for nuclei (DAPI, blue), aHSC ( $\alpha$-SMA, green), and apoptosis (TUNEL, red), and merged images. (G) Hepatic mRNA levels of $\alpha$-SMA, type I collagen, MMP1, and TIMP1 were measured by RT-PCR. Data represent the mean \pm SD of values per group. ${ }^{\star} P 0.05$ and ${ }^{* *} P 0.01$. UA, ursolic acid; ALT, alanine aminotransferase; AST, aspartate aminotransferase; TBIL, total bilirubin; TUNEL, TdT-mediated dUTP nick-end labeling; RT-PCR, real-time PCR. (H) $\alpha$-SMA, type I collagen, MMP1, and TIMP1 expression of proteins were detected by western blot.

GAPDH was the reference gene. The qRT-PCR primers are shown in Supplementary Table 1. The mRNA levels of type I collagen, MMP1, and TIMP1 were normalized to GAPDH mRNA levels.

\section{Statistical Analysis}

For the biochemical assays and histology score results, Image Pro Plus 6.0 software was used to check for normality. SPSS 23.0 software was used for data analysis. Image production and output were performed using GraphPad Prism 7.0 software. Each experiment was repeated three times to ensure confidence in the results. One-way analysis of variance (one-way ANOVA), Student's $t$ test, Mann-Whitney rank sum test, or Kruskal-Wallis $H$ test was used to analyze the significant differences between groups. $P<0.05$ was considered significant.

\section{RESULTS}

\section{UA Ameliorates Liver Injury and Fibrosis Induced by $\mathrm{CCl}_{4}$}

To clarify the effect of UA on the regression of $\mathrm{CCl}_{4}$-induced liver injury, we performed $\mathrm{H} \& \mathrm{E}$ and Masson's trichrome staining on liver sections (Figures 2A, B). The liver architecture of the control group was normal with intact lobule structure and with sinusoids and cords located in order around central veins. However, $\mathrm{CCl}_{4}$ treatment caused destruction of the normal structure, the formation of a fibrous septum, and formation of a pseudo-lobe, accompanied by inflammatory cell infiltration and hepatocellular necrosis in the liver of mice. This finding indicates the formation of liver fibrosis and inflammatory infiltration in mice of the $\mathrm{CCl}_{4}$ group. Compared with that in the $\mathrm{CCl}_{4}$ group, histological liver injury, disorder in the hepatic lobular structure, fibrous space, and collagen deposition in liver tissue and inflammatory cell infiltration were alleviated in the UA group. As shown in Figures 2C, D, quantitative analysis showed a significant improvement in the fibrotic score and area $(P<0.05)$. We also performed serum liver function tests to assess the liver damage (Figure 2E). ALT, AST, TBIL, and TG levels in mouse serum results indicated deterioration of liver function caused by $\mathrm{CCl}_{4}$ treatment but were partly restored by UA treatment.

Activated HSC leads to reprogramming of liver metabolism, increased autophagy, and increased parenchymal cell damage, leading to loss of HSC retinoids and increased contractility, and amplification of growth factors and inflammatory signaling factors in the liver microenvironment, which in turn generates a large amount of ECM to promote the occurrence and development of fibrosis (Lee et al., 2015). Therefore, we investigated the effect of UA on activated HSCs in vivo by dual-immunofluorescence staining for $\alpha$-SMA and TUNEL. As expected, $\alpha$-SMA-positive cells, a biomarker of activated HSCs (Iredale et al., 1998), and apoptosis in hepatocyte were pronounced observed in the $\mathrm{CCl}_{4}$ group compared to the control group. In contrast, $\alpha$-SMA-positive cells and apoptosis in hepatocyte were markedly decreased in the liver tissue of UA-treated mice (Figure 2F). Moreover, the change in the expression level of $a$-SMA also corresponded with the staining results $(P<0.05)$ (Figures $2 \mathrm{G}, \mathbf{H})$, thus clearly revealing that UA inhibits HSCs activation and reduces hepatocyte apoptosis in liver fibrosis mice.

The effect of UA on the expression of related factors involved in liver fibrosis in liver tissue was tested. At the mRNA level, $\mathrm{CCl}_{4}$ treatment induced high expression levels of type I collagen and TIMP1, but MMP1 promoted the degradation of the ECM and reduced fibrosis (Leroy et al., 2004), showing a decrease of expression. After UA intervention, the expression of type I collagen and TIMP1 genes was reduced (Figure 2G). At the protein level, results similar to the mRNA expression level results were observed. Type I collagen and TIMP1 showed increases in mice in the $\mathrm{CCl}_{4}$ group. The expression of these proteins was lower in the UA group than in the $\mathrm{CCl}_{4}$ group (Figure $2 \mathbf{H}$ ).

\section{UA Improves Intestinal Injury and Integrity in Liver Fibrosis Mice}

Intestinal damage and destruction of intestinal barrier integrity are often accompanied by liver fibrosis; therefore, we studied the improvement of intestinal function by UA. Histological changes and inflammatory conditions in all groups were assessed by $\mathrm{H} \& \mathrm{E}$ staining (Figure 3A). The orderly uniformly distributed villi and intestinal glands were aligned in the ilea of the control group. After $\mathrm{CCl}_{4}$ treatment, the villi were disordered and fragmented, and gland lumens were larger than those in the control group. However, in contrast to the $\mathrm{CCl}_{4}$ group, the intestinal villi of the mice in the UA group were more orderly and regular (Figure 3C). We used IHC to detect the proinflammatory factor TNF- $\alpha$ in ileal tissue (Figure 3B). The expression of TNF- $\alpha$ in ileal intestinal epithelial cells was significantly increased in the $\mathrm{CCl}_{4}$ group. After UA treatment, the expression of TNF- $\alpha$ decreased in the intestinal epithelial cells. The expression of TNF- $\alpha$ was also tested in the intestinal tissue. The expression level of TNF- $\alpha$ increased in $\mathrm{CCl}_{4}$ treated mice. Compared to the $\mathrm{CCl}_{4}$ group, the level of TNF- $\alpha$ in the ileum of the mice in the UA group was decreased $(P<0.01)$ (Figure 3D). These results indicate that UA can significantly improve the damaged intestine and inhibit inflammation.

Destruction of the intestinal barrier is another important intestinal change in liver fibrosis. Intestinal barrier integrity 


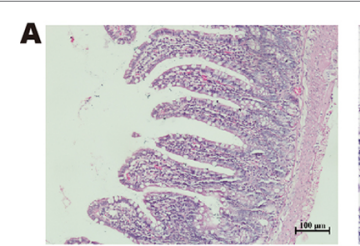

B

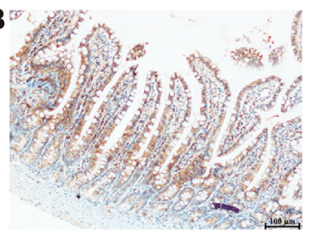

Control
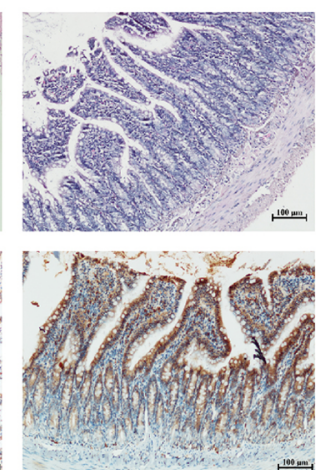

CCI4
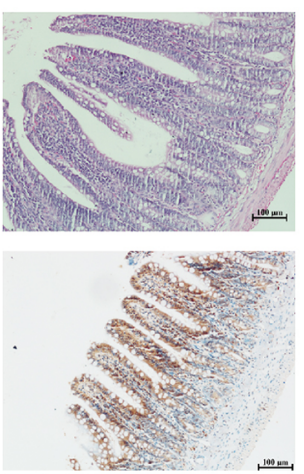

UA
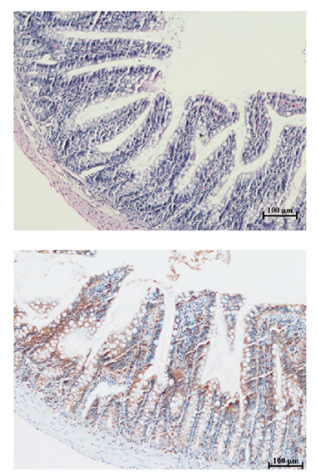

RhoAi
C

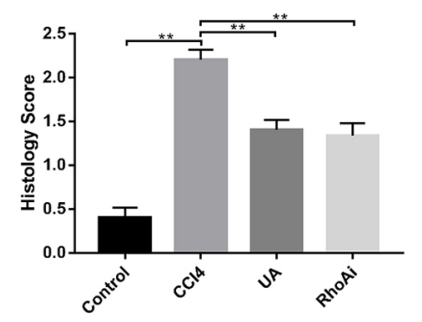

F

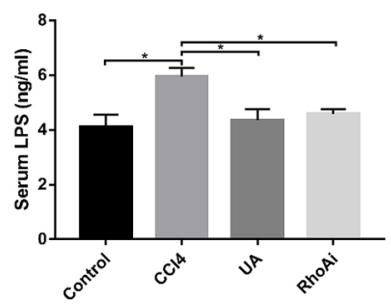

D

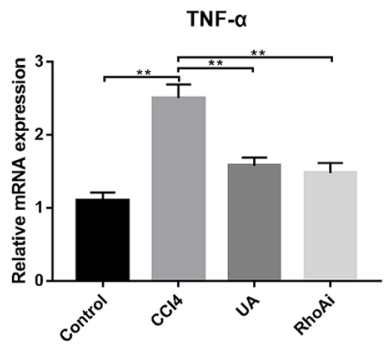

E
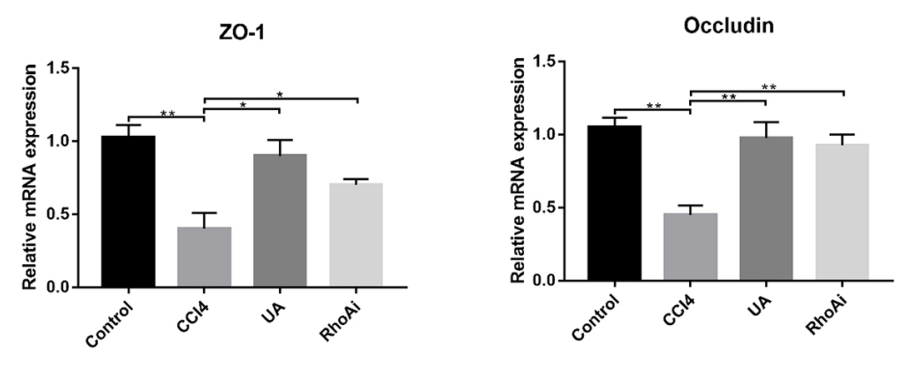

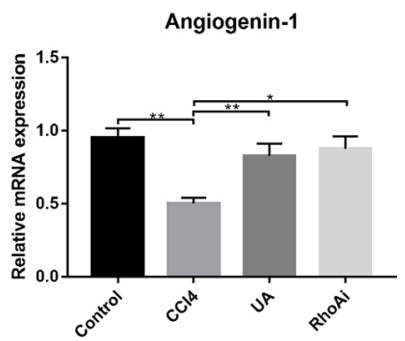

FIGURE 3 | Intestinal damage in $\mathrm{CCl}_{4}$-induced liver fibrosis mice is attenuated by UA. (A) H\&E staining (100x magnification). (B) TNF- $\alpha$ expression was measured via immunohistochemistry (IHC) staining (100× magnification). (C) H\&E staining intensity was quantified by histomorphometry. (D) lleal mRNA levels of TNF- $\alpha$ were measured by real-time RT-PCR. (E) lleal mRNA levels of tight junction (TJ) proteins, ZO-1 and occludin, and intestinal antimicrobial peptides angiogenin-1 were measured by RT-PCR. (F) Serum LPS levels were determined by ELISA analysis. Data represent the mean \pm SD of values per group. ${ }^{\star} P 0.05$ and ${ }^{\star \star} P 0.01$. UA, ursolic acid; RT-PCR, real-time PCR; LPS, lipopolysaccharide; ELISA, enzyme-linked immunosorbent assay.

is essential to resisting bacterial translocation and protecting the liver through the gut-liver axis (Cani and Delzenne, 2011; Schnabl and Brenner, 2014; Odenwald and Turner, 2017). Existing research has confirmed that tight junction (TJ) proteins and antibacterial peptide expression are inhibited by abnormal bile acid metabolism in fibrotic animals (Ubeda et al., 2016). Therefore, TJ proteins and antibacterial peptides in intestinal tissue were detected as important markers 
of the intestinal barrier. We speculated that UA protects the intestinal barrier by enhancing these connections. To verify this hypothesis, we explored the expression of the TJ proteins $\mathrm{ZO}-1$ and occludin, and intestinal antimicrobial peptides, angiogenin-1. The expression of both TJ proteins and antimicrobial peptides was lower in the mice of the $\mathrm{CCl}_{4}$ group. In fibrotic mice treated with $\mathrm{UA}$, the reduced level of ZO-1 and occludin mRNA expression was significantly restored. The protein expression levels in the ileum were similar to those of gene expression levels (Figure 3E). At the same time as the intestinal barrier is destroyed, the intestinal permeability is increased, and endotoxins enter the blood. Therefore, we measured the level of LPS in the serum. The level of LPS in the serum increased in the $\mathrm{CCl}_{4}$ group, which indicated that the intestinal permeability had increased in the $\mathrm{CCl}_{4}$-induced fibrotic mice. However, treatment with the UA combination reduced the LPS level in the intestines of liver fibrosis mice $(P<0.01)$ (Figure $3 \mathbf{F})$, confirming the protective effect of UA and strengthening of the intestinal barrier to reduce intestinal permeation.

\section{UA Ameliorates Microbiome Dysbiosis in Mice With Liver Fibrosis}

Liver cirrhosis often affects intestinal microbiota. Here, we explored the changes in intestinal microbiota in a liver fibrosis mouse model and the improvement effect of UA. We investigated the change of microbiota by next-generation sequencing in all groups. First, we assessed some indices used to represent the variation in the diversity and abundance of the microbiota (Figure 4A). The value of the Shannon index, the estimated microbial diversity, was significantly lower in the $\mathrm{CCl}_{4}$ group than in the control group $(P<0.01)$. Compared with the $\mathrm{CCl}_{4}$ group, UA significantly restored this reduced value of the Shannon index and increased the diversity $(P<0.01)$. Similarly, the overall microbial abundance was significantly elevated in mice treated with UA compared with $\mathrm{CCl}_{4}$-induced mice based on the Chaol index $(P<0.01)$. Next, the principal coordinates analysis (PCoA) plot showed a shift in the overall gut microbiota between the control group, $\mathrm{CCl}_{4}$ group, and UA group; each group belonged to a different microbiota (Figure 4B). These results demonstrate the changes in the microbiota during liver fibrosis and the corrective effects of UA.

The composition of the intestinal flora also changed due to the influence of liver fibrosis. We measured the composition of the microbial community in mice of all groups. At the phylum level, $\mathrm{CCl}_{4}$-treated mice displayed a reduced OTU level of Firmicutes, which include beneficial bacteria such as Lactobacillus. Lactobacillus can protect the body in multiple ways (Malik et al., 2018; Riehl et al., 2018; Caminero et al., 2019) (Figure 4C). However, UA ameliorated the distinctive enteric microbiome of mice with liver fibrosis. Compared with the $\mathrm{CCl}_{4}$ group, the UA-treated liver fibrosis mice showed higher OTUs of Firmicutes $(P<0.05)$, especially Lactobacillus. In addition, UA also had a similar improvement on bacterial community abundance at the genus level, including on Bifidobacterium and Lachnospiraceae (Figure 4D). These bacteria also serve to maintain health (Nicolucci et al., 2017; Schroeder et al., 2018). Next, the linear discriminant analysis effect size (LEfSe) was used to analyze the composition of the microbiota, and similar results were obtained. LEfSe showed that compared to the $\mathrm{CCl}_{4}$ group, the phylum Actinobacteria and the genera Bifidobacterium and Ruminiclostridium were enriched in the UA group (Figures 4E, F). We also predicted the function of the microbiome through the COG and KEGG databases (Supplementary Figure 2). The enriched pathways of the microbiome in $\mathrm{CCl}_{4}$-treated mice were as follows: "lipid transport and metabolism," "signal transduction mechanisms," "infectious diseases," "xenobiotics biodegradation and metabolism," and "metabolism of terpenoids and polyketides." However, after liver fibrosis mice were treated with UA, the enriched pathways of the microbiome changed to the following pathways: "carbohydrate transport and metabolism," "cell growth and death," and "enzyme families."

\section{UA Downregulates the RhoA Pathway, Improving Intestinal Damage and Gut Barrier Function}

As a molecule closely related to intestinal function and structure, RhoA plays an important role in intestinal damage, barrier destruction, and inflammation (Segain et al., 2003; LopezPosadas et al., 2016; Lopez-Posadas et al., 2017). We examined the ileal RhoA signaling pathway in liver fibrosis mice and the changes caused by UA. The mRNA expression levels of RhoA and ROCK1, the downstream target of RhoA, were upregulated in the ileal of mice in the $\mathrm{CCl}_{4}$ group $(P<0.01)$ (Figure 5A). Similarly, the protein expression levels of RhoA/ROCK pathway components were significantly higher in the ilea of mice in the $\mathrm{CCl}_{4}$ group than in the control group (Figures 5B, C). To better determine whether UA plays a role in intestinal protection through the regulation of RhoA, we inhibited RhoA in mice via tail vein AAV virus injection. In the RhoAi group, the histological damage, inflammatory cell infiltration, and TNF- $\alpha$ expression in the ilea were significantly decreased compared with the $\mathrm{CCl}_{4}$ group (Figures 3A-D). Furthermore, in RhoA-inhibited liver fibrosis mice, the destruction of the intestinal barrier integrity was also decreased $(P<0.05)$ (Figure 3E). Compared to the $\mathrm{CCl}_{4}$ group, the serum level of LPS was decreased in the RhoAi group, indicating the importance of RhoA in intestinal damage (Figure 3F).

We also studied the effect of UA on the expression of RhoA/ ROCK1. UA treatment significantly decreased the induced ileal expression level of RhoA and ROCK in liver fibrosis mice $(P<$ 0.01) (Figures 5A-C). From these results, we deduced that RhoA was probably the target of UA for intestinal protection.

\section{UA May Partially Improve Microbiome Dysbiosis Through RhoA}

To explore whether UA corrects the microbiome dysbiosis and has a close relationship with RhoA, we compared the changes in the composition and proportion of intestinal microbiota 
A
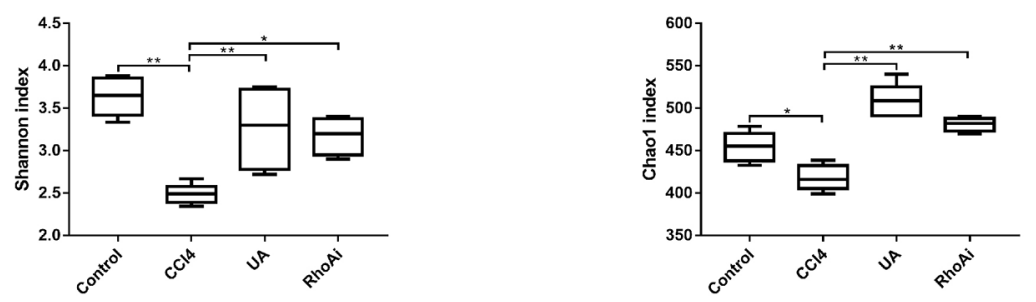

B

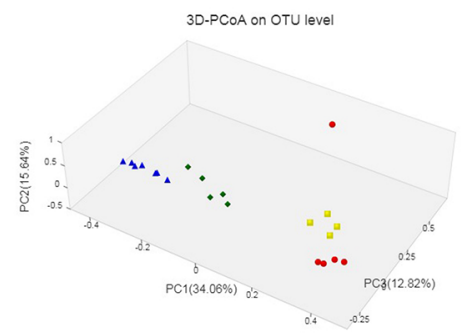

C

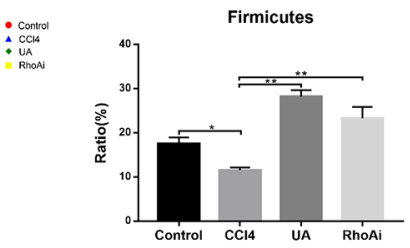

D

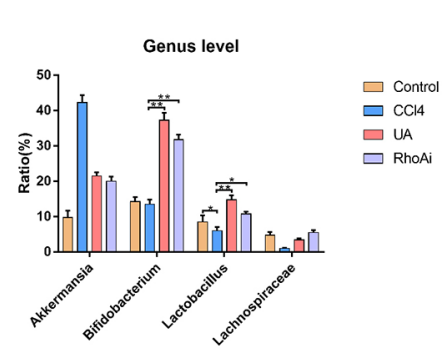

E
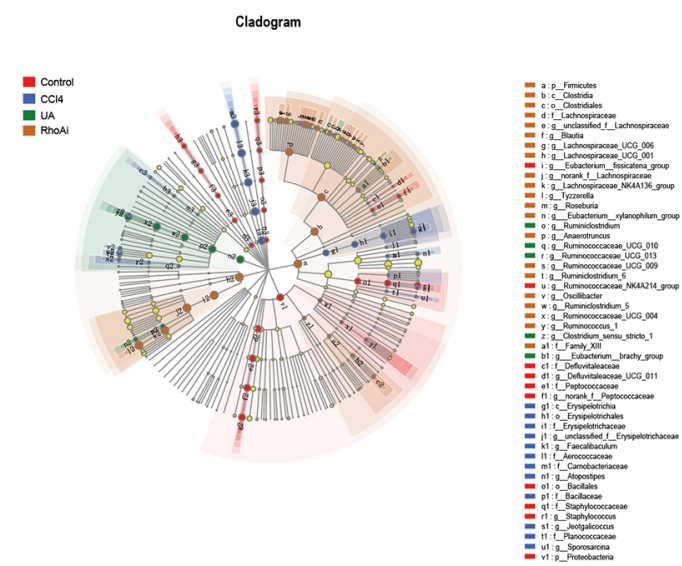

F
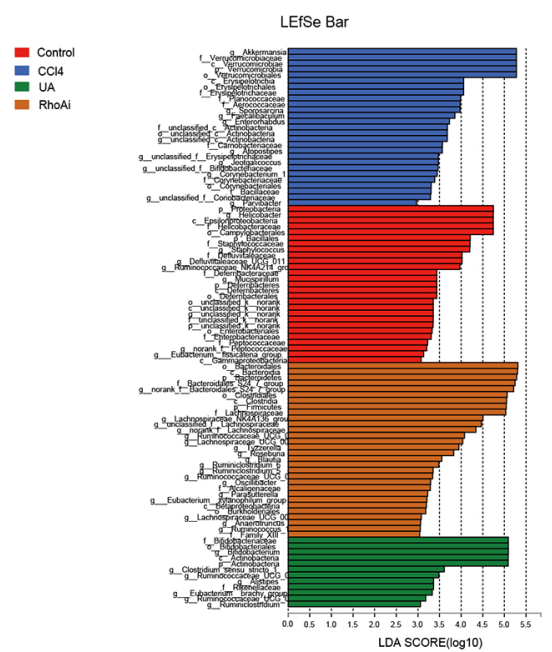

FIGURE 4 | Effect of UA on intestinal microbiota dysbiosis in $\mathrm{CCl}_{4}$-induced liver fibrosis. (A) The alpha diversity of each group was obtained using the Shannon index and the Chao1 index. (B) Principal coordinates analysis (PCoA) for the weighted UniFrac distance of the intestinal microbiota. (C) At the phylum level, changes in Firmicutes in mice of each group. (D) At the genus level, changes in microbiota in mice in each group. (E) Linear discriminant analysis effect size (LEfse) prediction was used to identify the most differentially abundant bacteria in each group. (F) LDA scores showed significant bacterial differences in each group. Only the bacteria meeting a significant LDA threshold value of 2 are shown. Data represent the mean \pm SD of values per group. ${ }^{*} P .05$ and ${ }^{* *} P 0.01$. UA, ursolic acid; LDA, linear discriminant analysis. 


\section{A}

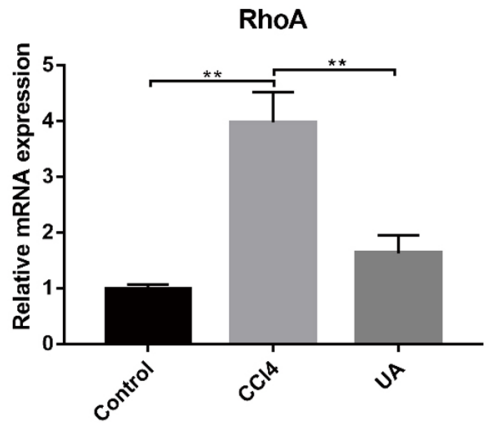

ROCK1

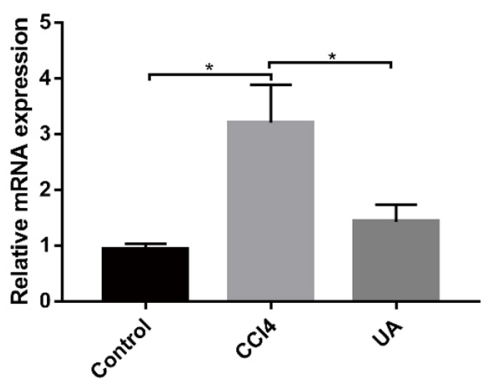

B

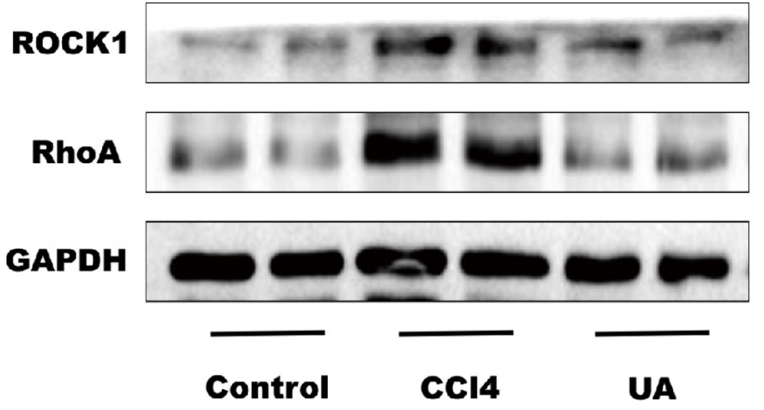

160KD

22KD

37KD

C
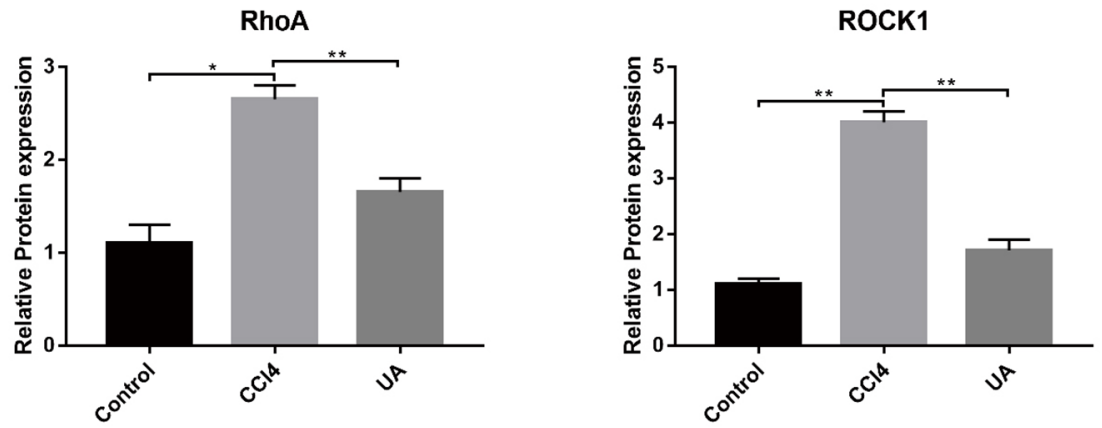

FIGURE 5 | Effect of UA on the RhoA-related pathway in the ileum of liver fibrosis mice. (A) lleal mRNA levels for RhoA and ROCK1 were measured by RT-PCR. (B) RhoA and ROCK1 expression of proteins was detected by western blot. (C) Histogram analysis of the levels of RhoA and ROCK1. Data represent the mean \pm SD of values per group. ${ }^{\star} P 0.05$ and ${ }^{\star *} P 0.01$. UA, ursolic acid; RT-PCR, real-time PCR.

of the UA group and RhoAi group. The Shannon and Chaol indexes, reflecting the diversity and abundance of microbiota, in mice of the RhoAi group did not statistically significantly change compared to those of the UA group (Figure 4A). The PCoA plot showed that the composition of the microbiome was different between the groups. The plot indicated that the microbiota of mice in the RhoAi group was similar to that of the control group (Figure 4B). Moreover, an increased abundance of Firmicutes was found in RhoA-inhibited fibrotic mice. The abundance of beneficial bacteria, such as Bifidobacterium and Lactobacillus, in the RhoAi group was higher than that in the $\mathrm{CCl}_{4}$ group, although lower than the UA group (Figures 4C, D). Interestingly, LEfSe indicated that the RhoAi group was characterized by a preponderance of the phylum Bacteroidetes and the order Clostridiales, which is different from that of the UA group (Figures 4E, F). In addition, the results of microbiome function prediction showed that enriched pathways of the microbiome in the RhoAi group were as follows: "cell motility," "transcription," "carbohydrate metabolism," and "immune system" (Supplementary Figure 2). We speculate that UA ameliorates the microbiota imbalance, which seems to partially occur through the regulation of RhoA. 


\section{DISCUSSION}

In this study, we show that UA, a traditional Chinese medicine administered to liver fibrosis, markedly reduces intestinal injury and destruction of the intestinal barrier and improves intestinal dysbiosis. We explored the mechanisms involved in this effect, speculating that UA-induced intestinal and microbiota improvements may be achieved via RhoA-related signaling pathways.

Liver fibrosis is a pathophysiological process that often occurs in chronic liver disease. The continued development of liver fibrosis can eventually develop into end-stage liver disease. It has been reported that liver fibrosis is often prone to intestinal damage and destruction of the intestinal barrier in patients and animal models (Parlesak et al., 2000; Hartmann et al., 2012; Du Plessis et al., 2013). Our results confirmed that intestinal villi become sparse and damaged in fibrotic mice. The expression of inflammation-related factors such as TNF- $\alpha$ also increased, synergistically destroying the intestinal tract. TJ proteins, which are markers of intestinal barrier integrity, are important for the maintenance of intestinal integrity (Zeisel et al., 2018) and showed decreased expression in $\mathrm{CCl}_{4}$-treated mice. In this situation, intestinal pathogenic factors are more likely to enter the bloodstream and return to the portal vein, which may accelerate the development of liver fibrosis.

Recently, a series of studies have found that disorders of microbiota occur in many liver diseases, including alcoholic liver disease, non-alcoholic fatty liver disease, primary sclerosing cholangitis, and liver cancer (Mouzaki et al., 2013; Szabo, 2015; Yu and Schwabe, 2017; Tedesco et al., 2018). Patients with liver cirrhosis often have bacterial disturbances (Chen et al., 2011). Cirrhosis modifies bile acid composition and causes its metabolic disorders, as observed in patients, and in an animal model of cirrhosis induced by $\mathrm{CCl}_{4}$ (Lorenzo-Zuniga et al., 2003; Kakiyama et al., 2014). The intestinal microbiota is regulated by the bacteriostatic properties of bile acids, including the synthesis of antimicrobial peptides and modulation of innate immunity (Inagaki et al., 2006). However, there are relatively few studies on the changes in intestinal microbiota in response to liver fibrosis. Therefore, we sequenced the fecal microbiota of liver fibrosis mice through $16 \mathrm{~S}$ rRNA technology. Our results are consistent with those of other studies in which the microbiota was found to be disordered in experimentally induced liver fibrosis animals (Wiest and Garcia-Tsao, 2005; Teltschik et al., 2012). By comparing the alpha diversity, Shannon and Chaol indexes of the bacteria, we found a significant decrease in the diversity and abundance of mice in the $\mathrm{CCl}_{4}$-induced liver fibrosis group. The PCoA plot showed that the microbiota of the control group and the $\mathrm{CCl}_{4}$ group belonged to two different communities. Moreover, we also analyzed the composition of the mouse microbiome in each group. Firmicutes, a phylum of bacteria that has been proven beneficial to the body (Warner and Lolkema, 2003; Reijnders et al., 2016; La Rosa et al., 2019), appeared to decrease in the $\mathrm{CCl}_{4}$ group compared to the control group. Other beneficial bacteria showed similar changes at the genus level, such as Lactobacillus and Bifidobacterium. Interestingly, Proteobacteria, especially Escherichia coli, did not show significant changes between groups. The possible reason is that not all bacteria change dramatically in the early stage of liver fibrosis, and some bacteria may change with the increase of liver fibrosis. The dysfunctional microbiota can stimulate intrahepatic inflammation and LPS-induced toll-like receptor (TLR)-related pathway activation through the damaged intestinal barrier, aggravating liver fibrosis (Soares et al., 2010; Zhu et al., 2012).

Our previous research confirmed that UA can alleviate liver fibrosis in animal models (Gan et al., 2018). The mechanism may involve UA inhibiting HSC activation by mediating NOX4related signaling pathways. Intestinal damage and bacterial dysbiosis are concomitant gut symptoms of liver fibrosis; however, the influence of UA on this is unknown. Therefore, we studied the effects of UA on the intestine and bacteria in liver fibrosis mice. In response to UA, intestinal damage was reduced, and intestinal barrier integrity was partially repaired in mice with liver fibrosis. Moreover, UA treatment restored damaged intestinal villi and inhibited the release of inflammatory factors. TJ proteins and antibacterial peptide expression showed some degree of recovery in the UA-treatment group. This recovery is significant for strengthening the intestinal barrier, which can prevent intestinal contaminants from flowing back into the liver through the portal vein.

A remarkable finding of our study was that UA can correct the intestinal microbiota imbalance in liver fibrosis mice. Previously, there has been little research on the effect of UA on the bacteria in liver fibrosis. We explored the improvement effect of UA on intestinal microbiota dysbiosis in $\mathrm{CCl}_{4}$-induced mice. After UA treatment, microbiota diversity and abundance partially recovered in liver fibrosis mice. Moreover, beneficial bacteria, including the phylum Firmicutes and the genera Lactobacillus and Bifidobacterium, rebounded in the UA group compared to the $\mathrm{CCl}_{4}$ group. These results confirm our hypothesis that UA restores microbiota stability.

RhoA, involved in cell migration, may be an important target for UA to improve intestinal damage and intestinal microbiota disorder. RhoA-related signaling pathways can induce TJ protein rearrangement and increase permeability by regulating myosin and actin, ultimately leading to the destruction of intestinal integrity (McKenzie and Ridley, 2007). Therefore, we used an AAV to interfere with the RhoA expression in a group of mice injected through the tail vein. Compared with the $\mathrm{CCl}_{4}$ treated mice, AAV-injected fibrotic mice had more less damage to the intestinal barrier and the intestinal microbiota, and the UA could inhibit the expression of RhoA pathway. These results indicate that the protective function of UA on the intestine is partly achieved by the inhibition of RhoA. It is worth noting that few studies have previously pointed out the link between RhoA and bacteria. In our study, we found that in RhoA-inhibited liver fibrosis mice, bacterial disorder was partly rescued. The mechanism involved may be that UA maintains the normal function of the intestinal mucosa by inhibiting RhoA and thus plays a role in correcting the disordered bacteria. This finding proves that RhoA may play an important role in the effect of UA.

The beneficial effect of UA on intestinal barrier integrity with the resultant reduction in disordered gut bacteria 
translocation may be a potential mechanism for UA to improve liver fibrosis. Liver fibrosis is often accompanied by the transfer of the microbiota to the portal vein and subsequent activation of the intrahepatic immune defense mechanisms, which causes an inflammatory reaction and accelerates the progression of liver fibrosis (Paik et al., 2003; Roh and Seki, 2013; Wiest et al., 2014). Therefore, we assume that UA prevents the bacteria from entering the vein by protecting the intestinal barrier, which reduces the immune response in the intestinal tract and the release of inflammatory factors, ultimately alleviating the development of liver fibrosis. This may be a potential mechanism by which UA exerts anti-fibrotic effects. However, the role of RhoA in intestinal microbiota dysbiosis and whether it is a target for UA intervention remain to be verified.

In conclusion, our research indicates that UA improves intestinal integrity and bacteria through RhoA-related signaling pathways, which provides strong evidence that UA indirectly contributes to the improvement of liver fibrosis through the gut-liver axis. The current study potentially provides new insights into the prevention and treatment of liver fibrosis by UA. However, the molecular mechanisms by which UA affects the gut and microbiota and the interaction with liver fibrosis need to be further explored. In combination with our previous research results, the findings herein provide theoretical support for the future use of UA in clinical practice.

\section{DATA AVAILABILITY STATEMENT}

All datasets generated for this study are included in the article/ Supplementary Material.

\section{REFERENCES}

Affo, S., Yu, L. X., and Schwabe, R. F. (2017). The role of cancer-associated fibroblasts and fibrosis in liver cancer. Annu. Rev. Pathol. 12, 153-186. doi: 10.1146/annurev-pathol-052016-100322

Akira, S., Uematsu, S., and Takeuchi, O. (2006). Pathogen recognition and innate immunity. Cell 124 (4), 783-801. doi: 10.1016/j.cell.2006.02.015

Asanuma, K., Yanagida-Asanuma, E., Faul, C., Tomino, Y., Kim, K., and Mundel, P. (2006). Synaptopodin orchestrates actin organization and cell motility via regulation of RhoA signalling. Nat. Cell Biol. 8 (5), 485-491. doi: 10.1038/ncb1400

Assimakopoulos, S. F., Tsamandas, A. C., Tsiaoussis, G. I., Karatza, E., Triantos, C., Vagianos, C. E., et al. (2012). Altered intestinal tight junctions' expression in patients with liver cirrhosis: a pathogenetic mechanism of intestinal hyperpermeability. Eur. J. Clin. Invest. 42 (4), 439-446. doi: 10.1111/j. 1365-2362.2011.02609.x

Caminero, A., McCarville, J. L., Zevallos, V. F., Pigrau, M., Yu, X. B., Jury, J., et al. (2019). Lactobacilli degrade wheat amylase trypsin inhibitors to reduce intestinal dysfunction induced by immunogenic wheat proteins. Gastroenterology 156 (8), 2266-2280.doi: 10.1053/j.gastro.2019.02.028

Cani, P. D., and Delzenne, N. M. (2011). The gut microbiome as therapeutic target. Pharmacol. Ther. 130 (2), 202-212. doi: 10.1016/j.pharmthera.2011.01.012

Cargnin, S. T., and Gnoatto, S. B. (2017). Ursolic acid from apple pomace and traditional plants: A valuable triterpenoid with functional properties. Food Chem. 220, 477-489. doi: 10.1016/j.foodchem.2016.10.029

Chen, Y., Yang, F., Lu, H., Wang, B., Chen, Y., Lei, D., et al. (2011). Characterization of fecal microbial communities in patients with liver cirrhosis. Hepatol. (Baltimore Md) 54 (2), 562-572. doi: 10.1002/hep.24423

\section{ETHICS STATEMENT}

The animal study was reviewed and approved by Institutional Animal Care and Use Committee of the First Affiliated Hospital of Nanchang University (Nanchang, China).

\section{AUTHOR CONTRIBUTIONS}

S-ZW and CL contributed equally to this study. S-ZW and CL designed and wrote the manuscript. $\mathrm{C}-\mathrm{KH}$ and F-YL analyzed data. XZ critically revised the manuscript.

\section{FUNDING}

This study was supported by the National Natural Science Foundation of China (grant number 81660110), the "Gan-Po Talent 555" Project of Jiangxi Province, and the Nanchang University Graduate Innovation Special Fund Project (grant number CX2018205).

\section{ACKNOWLEDGMENTS}

We would like to thank the National Natural Science Foundation of China for the economic support.

\section{SUPPLEMENTARY MATERIAL}

The Supplementary Material for this article can be found online at: https://www.frontiersin.org/articles/10.3389/fphar.2019.01321/ full\#supplementary-material

Chiu, C. J., McArdle, A. H., Brown, R., Scott, H. J., and Gurd, F. N. (1970) Intestinal mucosal lesion in low-flow states. I. A morphological, hemodynamic, and metabolic reappraisal. Arch. Surg. 101 (4), 478-483. doi: 10.1001/ archsurg.1970.01340280030009

Citalan-Madrid, A. F., Vargas-Robles, H., Garcia-Ponce, A., Shibayama, M., Betanzos, A., Nava, P., et al. (2017). Cortactin deficiency causes increased RhoA/ROCK1-dependent actomyosin contractility, intestinal epithelial barrier dysfunction, and disproportionately severe DSS-induced colitis. Mucosal Immunol. 10 (5), 1237-1247. doi: 10.1038/mi.2016.136

Claesson, M.J., Jeffery, I.B., Conde, S., Power, S.E., O'Connor, E.M., Cusack, S., et al. (2012). Gut microbiota composition correlates with diet and health in the elderly. Nature 488 (7410), 178-184. doi: 10.1038/nature11319

Doonan, J., Tarafdar, A., Pineda, M. A., Lumb, F. E., Crowe, J., Khan, A. M., et al. (2019). The parasitic worm product ES-62 normalises the gut microbiota bone marrow axis in inflammatory arthritis. Nat. Commun. 10 (1), 1554. doi: 10.1038/s41467-019-09361-0

Du Plessis, J., Vanheel, H., Janssen, C. E., Roos, L., Slavik, T., Stivaktas, P. I., et al. (2013). Activated intestinal macrophages in patients with cirrhosis release NO and IL-6 that may disrupt intestinal barrier function. J. Hepatol. 58 (6), 11251132. doi: 10.1016/j.jhep.2013.01.038

Fouts, D. E., Torralba, M., Nelson, K. E., Brenner, D. A., and Schnabl, B. (2012). Bacterial translocation and changes in the intestinal microbiome in mouse models of liver disease. J. Hepatol. 56 (6), 1283-1292. doi: 10.1016/j. jhep.2012.01.019

Gan, D., Zhang, W., Huang, C., Chen, J., He, W., Wang, A., et al. (2018). Ursolic acid ameliorates CCl4-induced liver fibrosis through the NOXs/ROS pathway. J. Cell Physiol. 233 (10), 6799-6813. doi: 10.1002/jcp.26541 
Garidou, L., Pomie, C., Klopp, P., Waget, A., Charpentier, J., Aloulou, M., et al. (2015). The gut microbiota regulates intestinal CD4 $\mathrm{T}$ cells expressing RORgammat and controls metabolic disease. Cell Metab. 22 (1), 100-112. doi: 10.1016/j.cmet.2015.06.001

Hartmann, P., Haimerl, M., Mazagova, M., Brenner, D. A., and Schnabl, B. (2012). Toll-like receptor 2-mediated intestinal injury and enteric tumor necrosis factor receptor I contribute to liver fibrosis in mice. Gastroenterology 143 (5), 1330-40 e1. doi: 10.1053/j.gastro.2012.07.099

He, W., Shi, F., Zhou, Z. W., Li, B., Zhang, K., Zhang, X., et al. (2015). A bioinformatic and mechanistic study elicits the antifibrotic effect of ursolic acid through the attenuation of oxidative stress with the involvement of ERK, PI3K/Akt, and p38 MAPK signaling pathways in human hepatic stellate cells and rat liver. Drug Des. Devel. Ther. 9, 3989-4104. doi: 10.2147/ DDDT.S85426

Ikeda, Y., Murakami, A., Fujimura, Y., Tachibana, H., Yamada, K., Masuda, D., et al. (2007). Aggregated ursolic acid, a natural triterpenoid, induces IL-1beta release from murine peritoneal macrophages: role of CD36. J. Immunol. 178 (8), 4854-4864. doi: 10.4049/jimmunol.178.8.4854.

Inagaki, T., Moschetta, A., Lee, Y. K., Peng, L., Zhao, G., Downes, M., et al. (2006). Regulation of antibacterial defense in the small intestine by the nuclear bile acid receptor. Proc. Natl. Acad. Sci. U.S.A. 103 (10), 3920-3925. doi: 10.1073/ pnas.0509592103

Iredale, J. P., Benyon, R. C., Pickering, J., McCullen, M., Northrop, M., Pawley, S., et al. (1998). Mechanisms of spontaneous resolution of rat liver fibrosis. Hepatic stellate cell apoptosis and reduced hepatic expression of metalloproteinase inhibitors. J. Clin. Invest. 102 (3), 538-549. doi: 10.1172/JCI1018

Jie, Z., Xia, H., Zhong, S. L., Feng, Q., Li, S., Liang, S., et al. (2017). The gut microbiome in atherosclerotic cardiovascular disease. Nat. Commun. 8 (1), 845. doi: 10.1038/s41467-017-00900-1

Kakiyama, G., Hylemon, P. B., Zhou, H., Pandak, W. M., Heuman, D. M., Kang, D. J., et al. (2014). Colonic inflammation and secondary bile acids in alcoholic cirrhosis. Am. J. Physiol. Gastrointest. Liver Physiol. 306 (11), G929-G937. doi: 10.1152/ajpgi.00315.2013

Kalaitzakis, E. (2014). Gastrointestinal dysfunction in liver cirrhosis. World J. Gastroenterol. 20 (40), 14686-14695. doi: 10.3748/wjg.v20.i40.14686

Kim, J. S., Kim, J. G., Moon, M. Y., Jeon, C. Y., Won, H. Y., Kim, H. J., et al. (2006). Transforming growth factor-betal regulates macrophage migration via RhoA. Blood 108 (6), 1821-1829. doi: 10.1182/blood-2005-10-009191

Kim, M. H., Kim, J. N., Han, S. N., and Kim, H. K. (2015). Ursolic acid isolated from guava leaves inhibits inflammatory mediators and reactive oxygen species in LPS-stimulated macrophages. Immunopharmacol. Immunotoxicol. 37 (3), 228-235. doi: 10.3109/08923973.2015.1021355

Kumar, H., Kawai, T., and Akira, S. (2011). Pathogen recognition by the innate immune system. Int. Rev. Immunol. 30 (1), 16-34. doi: $10.3109 / 08830185.2010 .529976$

La Rosa, S. L., Leth, M. L., Michalak, L., Hansen, M. E., Pudlo, N. A., Glowacki, R., et al. (2019). The human gut Firmicute Roseburia intestinalis is a primary degrader of dietary beta-mannans. Nat. Commun. 10 (1), 905. doi: 10.1038/ s41467-019-08812-y

Lee, Y. A., Wallace, M. C., and Friedman, S. L. (2015). Pathobiology of liver fibrosis: a translational success story. Gut 64 (5), 830-841. doi: 10.1136/ gutjnl-2014-306842

Leroy, V., Monier, F., Bottari, S., Trocme, C., Sturm, N., Hilleret, M. N., et al. (2004). Circulating matrix metalloproteinases 1, 2, 9 and their inhibitors TIMP-1 and TIMP-2 as serum markers of liver fibrosis in patients with chronic hepatitis C: comparison with PIIINP and hyaluronic acid. Am. J. Gastroenterol. 99 (2), 271-279.

Li, Z., Gao, M., Yang, B., Zhang, H., Wang, K., Liu, Z., et al. (2018). Naringin attenuates MLC phosphorylation and NF-kappaB activation to protect sepsisinduced intestinal injury via RhoA/ROCK pathway. BioMed. Pharmacother. 103, 50-58. doi: 10.1016/j.biopha.2018.03.163

Lopez-Posadas, R., Becker, C., Gunther, C., Tenzer, S., Amann, K., Billmeier, U., et al. (2016). Rho-A prenylation and signaling link epithelial homeostasis to intestinal inflammation. J. Clin. Invest. 126 (2), 611-626. doi: 10.1172/JCI80997

Lopez-Posadas, R., Sturzl, M., Atreya, I., Neurath, M. F., and Britzen-Laurent, N. (2017). Interplay of GTPases and Cytoskeleton in Cellular Barrier Defects during Gut Inflammation. Front. Immunol. 8, 1240. doi: 10.3389/ fimmu.2017.01240
Lorenzo-Zuniga, V., Bartoli, R., Planas, R., Hofmann, A. F., Vinado, B., Hagey, L. R., et al. (2003). Oral bile acids reduce bacterial overgrowth, bacterial translocation, and endotoxemia in cirrhotic rats. Hepatol. (Baltimore $M d$ ) 37 (3), 551-557. doi: 10.1053/jhep.2003.50116

Lozupone, C. A., Stombaugh, J. I., Gordon, J. I., Jansson, J. K., and Knight, R. (2012). Diversity, stability and resilience of the human gut microbiota. Nature 489 (7415), 220-230. doi: 10.1038/nature11550

Malik, M., Suboc, T. M., Tyagi, S., Salzman, N., Wang, J., Ying, R., et al. (2018). Lactobacillus plantarum 299v supplementation improves vascular endothelial function and reduces inflammatory biomarkers in men with stable coronary artery disease. Circ. Res. 123 (9), 1091-1102. doi: 10.1161/ CIRCRESAHA.118.313565

Maynard, C. L., Elson, C. O., Hatton, R. D., and Weaver, C. T. (2012). Reciprocal interactions of the intestinal microbiota and immune system. Nature 489 (7415), 231-241. doi: 10.1038/nature11551

McKenzie, J. A., and Ridley, A. J. (2007). Roles of Rho/ROCK and MLCK in TNFalpha-induced changes in endothelial morphology and permeability. J. Cell Physiol. 213 (1), 221-228. doi: 10.1002/jcp.21114

Mouzaki, M., Comelli, E. M., Arendt, B. M., Bonengel, J., Fung, S. K., Fischer, S. E., et al. (2013). Intestinal microbiota in patients with nonalcoholic fatty liver disease. Hepatol. (Baltimore Md) 58 (1), 120-127. doi: 10.1002/hep.26319

Nicolucci, A. C., Hume, M. P., Martinez, I., Mayengbam, S., Walter, J., and Reimer, R. A. (2017). Prebiotics reduce body fat and alter intestinal microbiota in children who are overweight or with obesity. Gastroenterology 153 (3), 711722. doi: 10.1053/j.gastro.2017.05.055

Odenwald, M. A., and Turner, J. R. (2017). The intestinal epithelial barrier: a therapeutic target? Nat. Rev. Gastroenterol. Hepatol. 14 (1), 9-21. doi: 10.1038/ nrgastro.2016.169

Paik, Y. H., Schwabe, R. F., Bataller, R., Russo, M. P., Jobin, C., and Brenner, D. A. (2003). Toll-like receptor 4 mediates inflammatory signaling by bacterial lipopolysaccharide in human hepatic stellate cells. Hepatol. (Baltimore $\mathrm{Md}$ ) 37 (5), 1043-1055. doi: 10.1053/jhep.2003.50182

Palma, P., Mihaljevic, N., Hasenberg, T., Keese, M., and Koeppel, T. A. (2007). Intestinal barrier dysfunction in developing liver cirrhosis: an in vivo analysis of bacterial translocation. Hepatol. Res. 37 (1), 6-12. doi: 10.1111/j.1872-034X.2007.00004.x

Parlesak, A., Schafer, C., Schutz, T., Bode, J. C., and Bode, C. (2000). Increased intestinal permeability to macromolecules and endotoxemia in patients with chronic alcohol abuse in different stages of alcohol-induced liver disease. J. Hepatol. 32 (5), 742-747. doi: 10.1016/s0168-8278(00)80242-1

Poynard, T., Bedossa, P., and Opolon, P. (1997). Natural history of liver fibrosis progression in patients with chronic hepatitis C. The OBSVIRC, METAVIR, CLINIVIR, and DOSVIRC groups. Lancet 349 (9055), 825-832. doi: 10.1016/ s0140-6736(96)07642-8

Reijnders, D., Goossens, G. H., Hermes, G. D., Neis, E. P., van der Beek, C. M., Most, J., et al. (2016). Effects of gut microbiota manipulation by antibiotics on host metabolism in obese humans: a randomized double-blind placebocontrolled trial. Cell Metab. 24 (1), 63-74. doi: 10.1016/j.cmet.2016.06.016

Riehl, T. E., Alvarado, D., Ee, X., Zuckerman, A., Foster, L., Kapoor, V., et al. (2018). Lactobacillus rhamnosus GG protects the intestinal epithelium from radiation injury through release of lipoteichoic acid, macrophage activation and the migration of mesenchymal stem cells. Gut. 68 (6), 1003-1013. doi: 10.1136/ gutjnl-2018-316226

Roh, Y. S., and Seki, E. (2013). Toll-like receptors in alcoholic liver disease, nonalcoholic steatohepatitis and carcinogenesis. J. Gastroenterol. Hepatol. 28 Suppl 1, 38-42. doi: 10.1111/jgh.12019

Schnabl, B., and Brenner, D. A. (2014). Interactions between the intestinal microbiome and liver diseases. Gastroenterology 146 (6), 1513-1524. doi: 10.1053/j.gastro.2014.01.020

Schroeder, B. O., Birchenough, G. M. H., Stahlman, M., Arike, L., Johansson, M. E. V., Hansson, G. C., et al. (2018). Bifidobacteria or fiber protects against diet-induced microbiota-mediated colonic mucus deterioration. Cell Host Microbe 23 (1), 27-40 e7. doi: 10.1016/j.chom.2017.11.004

Segain, J. P., Raingeard de la Bletiere, D., Sauzeau, V., Bourreille, A., Hilaret, G., Cario-Toumaniantz, C., et al. (2003). Rho kinase blockade prevents inflammation via nuclear factor kappa B inhibition: evidence in Crohn's disease and experimental colitis. Gastroenterology 124 (5), 1180-1187. doi: 10.1016/ s0016-5085(03)00283-x. 
Seki, E., and Brenner, D. A. (2015). Recent advancement of molecular mechanisms of liver fibrosis. J. Hepatobiliary Pancreat. Sci. 22 (7), 512-518. doi: 10.1002/ jhbp. 245

Shackel, N. A., Vadas, M. A., Gamble, J. R., and McCaughan, G. W. (2014). Beyond liver fibrosis: hepatic stellate cell senescence links obesity to liver cancer by way of the microbiome. Hepatol. (Baltimore Md) 59 (6), 2413-2415. doi: 10.1002/ hep. 26932

Soares, J. B., Pimentel-Nunes, P., Roncon-Albuquerque, R., and Leite-Moreira, A. (2010). The role of lipopolysaccharide/toll-like receptor 4 signaling in chronic liver diseases. Hepatol. Int. 4 (4), 659-672. doi: 10.1007/s12072-010-9219-x

Szabo, G. (2015). Gut-liver axis in alcoholic liver disease. Gastroenterology 148 (1), 30-36. doi: 10.1053/j.gastro.2014.10.042

Tedesco, D., Thapa, M., Chin, C. Y., Ge, Y., Gong, M., Li, J., et al. (2018). Alterations in intestinal microbiota lead to production of interleukin 17 by intrahepatic gammadelta T-Cell receptor-positive cells and pathogenesis of cholestatic liver disease. Gastroenterology 154 (8), 2178-2193. doi: 10.1053/j.gastro.2018.02.019

Teltschik, Z., Wiest, R., Beisner, J., Nuding, S., Hofmann, C., Schoelmerich, J., et al. (2012). Intestinal bacterial translocation in rats with cirrhosis is related to compromised Paneth cell antimicrobial host defense. Hepatol. (Baltimore Md) 55 (4), 1154-1163. doi: 10.1002/hep.24789

Thompson, A. I., Conroy, K. P., and Henderson, N. C. (2015). Hepatic stellate cells: central modulators of hepatic carcinogenesis. BMC Gastroenterol. 15, 63. doi: 10.1186/s12876-015-0291-5

Ticinesi, A., Milani, C., Guerra, A., Allegri, F., Lauretani, F., Nouvenne, A., et al. (2018). Understanding the gut-kidney axis in nephrolithiasis: an analysis of the gut microbiota composition and functionality of stone formers. Gut 67 (12), 2097-2106. doi: 10.1136/gutjnl-2017-315734

Tong, J., Wang, Y., Chang, B., Zhang, D., and Wang, B. (2013). Evidence for the involvement of RhoA signaling in the ethanol-induced increase in intestinal epithelial barrier permeability. Int. J. Mol. Sci. 14 (2), 3946-3960. doi: 10.3390/ ijms 14023946

Tremaroli, V., and Backhed, F. (2012). Functional interactions between the gut microbiota and host metabolism. Nature 489 (7415), 242-249. doi: 10.1038/ nature 11552

Turnbaugh, P. J., Hamady, M., Yatsunenko, T., Cantarel, B. L., Duncan, A., Ley, R. E., et al. (2009). A core gut microbiome in obese and lean twins. Nature 457 (7228), 480-484. doi: 10.1038/nature07540

Ubeda, M., Lario, M., Munoz, L., Borrero, M. J., Rodriguez-Serrano, M., SanchezDiaz, A. M., et al. (2016). Obeticholic acid reduces bacterial translocation and inhibits intestinal inflammation in cirrhotic rats. J. Hepatol. 64 (5), 1049-1057. doi: 10.1016/j.jhep.2015.12.010

Wang, X., Ikejima, K., Kon, K., Arai, K., Aoyama, T., Okumura, K., et al. (2011). Ursolic acid ameliorates hepatic fibrosis in the rat by specific induction of apoptosis in hepatic stellate cells. J. Hepatol. 55 (2), 379-387. doi: 10.1016/j. jhep.2010.10.040

Warner, J. B., and Lolkema, J. S. (2003). CcpA-dependent carbon catabolite repression in bacteria. Microbiol. Mol. Biol. Rev. 67 (4), 475-490. doi: 10.1128/ mmbr.67.4.475-490.2003.

Wiest, R., and Garcia-Tsao, G. (2005). Bacterial translocation (BT) in cirrhosis. Hepatol. (Baltimore Md) 41 (3), 422-433. doi: 10.1002/hep.20632

Wiest, R., Lawson, M., and Geuking, M. (2014). Pathological bacterial translocation in liver cirrhosis. J. Hepatol. 60 (1), 197-209. doi: 10.1016/j.jhep. 2013.07.044

Wu, H. Y., Chang, C.I., Lin, B. W., Yu, F. L., Lin, P. Y., Hsu, J. L., et al. (2011). Suppression of hepatitis B virus $\mathrm{x}$ protein-mediated tumorigenic effects by ursolic Acid. J. Agric. Food Chem. 59 (5), 1713-1722. doi: 10.1021/jf1045624

Yu, L. X., and Schwabe, R. F. (2017). The gut microbiome and liver cancer: mechanisms and clinical translation. Nat. Rev. Gastroenterol. Hepatol. 14 (9), 527-539. doi: 10.1038/nrgastro.2017.72

Zeisel, M. B., Dhawan, P., and Baumert, T. F. (2018). Tight junction proteins in gastrointestinal and liver disease. Gut. 68 (3), 547-561. doi: 10.1136/ gutjnl-2018-316906

Zhu, Q., Zou, L., Jagavelu, K., Simonetto, D. A., Huebert, R. C., Jiang, Z. D., et al. (2012). Intestinal decontamination inhibits TLR4 dependent fibronectinmediated cross-talk between stellate cells and endothelial cells in liver fibrosis in mice. J. Hepatol. 56 (4), 893-899. doi: 10.1016/j.jhep.2011.11.013

Conflict of Interest: The authors declare that the research was conducted in the absence of any commercial or financial relationships that could be construed as a potential conflict of interest.

Copyright (C) 2019 Wan, Liu, Huang, Luo and Zhu. This is an open-access article distributed under the terms of the Creative Commons Attribution License (CC $B Y)$. The use, distribution or reproduction in other forums is permitted, provided the original author(s) and the copyright owner(s) are credited and that the original publication in this journal is cited, in accordance with accepted academic practice. No use, distribution or reproduction is permitted which does not comply with these terms. 